BNL-NT-06/21

\title{
THRESHOLD RESUMMATION EFFECTS IN THE POLARIZED DRELL-YAN PROCESS AT GSI AND J-PARC*
}

\author{
HIROSHI YOKOYA \\ Department of Physics, Niigata University, \\ Niigata 950-2181, Japan \\ E-mail: yokoya@nt.sc.niigata-u.ac.jp \\ WERNER VOGELSANG \\ Physics Department, Brookhaven National Laboratory, \\ Upton, New York 11973, U.S.A. \\ E-mail: vogelsan@quark.phy.bnl.gov
}

\begin{abstract}
We present studies of QCD corrections to dilepton production in transversely polarized $p p$ and $\bar{p} p$ scattering. In particular we briefly discuss the effects of NNLL threshold resummation on the rapidity distribution of the lepton pair.
\end{abstract}

\section{Introduction}

Recently, new experiments in polarized hadron collisions have been proposed at the $\operatorname{GSI}^{1}(\bar{p} p)$ and at $\mathrm{J}_{-\mathrm{PARC}}{ }^{2}(p p)$. One of the main purposes of these experiments is the measurement of transverse-spin asymmetries in the Drell-Yan process, in order to get information on the transversely polarized parton distribution functions (PDFs) of the nucleon. The proposed experiments would be at relatively modest collision energies, e.g. $\sqrt{S}=14.5 \mathrm{GeV}$ at GSI-PAX and $\sqrt{S}=10 \mathrm{GeV}$ at J-PARC. At these energies, perturbativeQCD (pQCD) corrections as well as power-suppressed contributions may be large and require careful theoretical study.

In this brief note, we report on recent studies of $\mathrm{pQCD}$ corrections to the invariant-mass and rapidity distributions of Drell-Yan pairs ${ }^{3}$. In particular, we consider the all-order resummation of large "threshold" logarithms ${ }^{4}$.

*Talk presented by H. Yokoya at the "XIV International Workshop on Deep Inelastic Scattering (DIS2006)", April 20-24, 2006, Tsukuba, Japan. 
Our aim is to see the behavior of QCD higher-order corrections in this kinematic regime, and to investigate the self-consistency of the pQCD framework. For further details, including a discussion of possible nonperturbative effects to the cross section, see $^{3}$.

\section{Mass Distributions}

The invariant-mass distribution of Drell-Yan lepton pairs can be written in terms of the PDFs and partonic hard-scattering cross sections as

$$
\frac{d \sigma}{d M^{2}}=N \sum_{a b} \int_{\tau}^{1} \frac{d x_{1}}{x_{1}} f_{a}\left(x_{1}, \mu\right) \int_{\tau / x_{1}}^{1} \frac{d x_{2}}{x_{2}} f_{b}\left(x_{2}, \mu\right) \omega_{a b}\left(z, \alpha_{s}(\mu), r\right) .
$$

The transversely polarized cross section is written in an analogous manner. In (1), $\tau=M^{2} / S, z=\tau / x_{1} x_{2}$ and $r=M^{2} / \mu^{2}$, with $\mu$ the renormalization/factorization scale. $N$ is defined so that the $\mathcal{O}\left(\alpha_{s}^{0}\right)$ term becomes

$\omega_{q \bar{q}}^{(0)}=\delta(1-z)$. The higher-order functions $\omega_{a b}^{(i)}$ have been calculated to $\mathcal{O}\left(\alpha_{s}^{2}\right)$ for the unpolarized cross section ${ }^{5}$, and to $\mathcal{O}\left(\alpha_{s}^{1}\right)$ for the transversely polarized one ${ }^{6}$.

The numerical size of the NNLO corrections for GSI or J-PARC kinematics amounts to more than three times the LO cross section at high $M$. It is known that these large corrections come from the threshold region where the partonic energy is just enough to produce the lepton pair of invariant mass $M$. In this region, large "threshold" logarithms arise. The systematic way of taking into account these logarithms to all orders, called "threshold resummation", has been developed in ${ }^{4}$. The resummation is achieved in Mellin-moment space, where it gives rise to a Sudakov exponent. Presently, the exponent for the Drell-Yan process is known to NNLL accuracy. Defining $\omega_{a b}(n)=\int_{0}^{1} d z z^{n-1} \omega_{a b}(z)$, one has:

$$
\omega_{q \bar{q}}^{\mathrm{res}}\left(n, \alpha_{s}, r\right)=C_{D Y}\left(\alpha_{s}, r\right) \exp \left[\frac{1}{\alpha_{s}} h_{q}^{(1)}(\lambda)+h_{q}^{(2)}(\lambda, r)+\alpha_{s} h_{q}^{(3)}(\lambda, r)\right],
$$

where $\lambda=b_{0} \alpha_{s} \ln n$. The detailed expressions for the $n$-independent coefficient $C_{D Y}$ and the functions $h_{q}^{(i)}$ may be found, e.g., in Ref. ${ }^{7}$. We note that $C_{D Y}$ is also known to exponentiate ${ }^{8}$. We use the "Minimal Prescription"9 for dealing with the Landau pole in the resummed expression.

It is known that the resummation formula can be improved to include collinear (non-soft) gluon effects ${ }^{10,11}$. In NLO, these correspond to terms $\propto \ln (n) / n$. They may be taken into account in the resummation by including certain subleading terms in the exponent, associated with DGLAP evolution of parton distributions. Through singlet mixing in evolution, these 

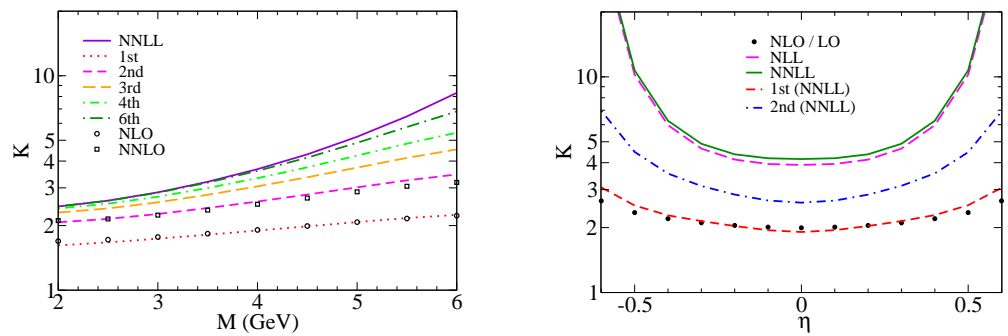

Figure 1. $K$-factors for the resummed cross section and its perturbative expansions for $p p$ collisions at $\sqrt{S}=10 \mathrm{GeV}$. Left: invariant-mass distribution, right: rapidity distribution at $M=5 \mathrm{GeV}$. The NLO (NNLO) $K$-factors are also plotted as circle (square) symbols.

subleading terms also feed into the $q g$-subprocess ${ }^{11}$. We found that these effects are significant, especially for the case of $p p$ collisions at J-PARC.

Fig. 1(left) shows the resummed $K$-factor for the J-PARC situation. Expansions of the resummed $K$-factor to fixed perturbative orders are also plotted. We stress that the second- and third-order expansions are in good agreement with the full NLO and NNLO results. This shows that the higher-order corrections are indeed dominated by the threshold logarithms, and that the resummation is accurately reproducing the latter.

\section{Resummation for Rapidity Distributions}

We now consider the cross section differential in the lepton pair's rapidity,

$$
\frac{d \sigma}{d M^{2} d \eta}=N \sum_{a b} \int_{x_{1}^{0}}^{1} d x_{1} f_{a}\left(x_{1}\right) \int_{x_{2}^{0}}^{1} d x_{2} f_{b}\left(x_{2}\right) D_{a b}\left(x_{1}^{0}, x_{2}^{0}, x_{1}, x_{2}, \alpha_{s}\right),
$$

where $x_{1,2}^{0}=\sqrt{\tau} \mathrm{e}^{ \pm \eta}$. The $D_{a b}$ have been calculated perturbatively up to $\mathcal{O}\left(\alpha_{s}^{2}\right)$ for unpolarized cross section ${ }^{12}$, and to $\mathcal{O}\left(\alpha_{s}\right)$ for the transversely polarized case ${ }^{13}$. The $\mathcal{O}\left(\alpha_{s}^{0}\right)$ term is simply $D_{q \bar{q}}^{(0)}=\delta\left(x_{1}-x_{1}^{0}\right) \delta\left(x_{2}-x_{2}^{0}\right)$. The application of the threshold resummation technique to rapidity distributions has been discussed e.g. in Ref. ${ }^{14}$. In addition to the usual Mellin transform in $\tau$, it makes use of a Fourier transform in $\eta$. The cross section in double-transform space can be written as

$$
\begin{aligned}
\tilde{\sigma}\left(n, m, \alpha_{s}, r\right) & \equiv \int_{0}^{1} d \tau \tau^{n-1} \int d \eta e^{i m \eta} \frac{d \sigma}{d M^{2} d \eta} \\
& =N \sum_{a b} f_{a}\left(n+\frac{i}{2} m\right) f_{b}\left(n-\frac{i}{2} m\right) \tilde{D}_{a b}\left(n, m, \alpha_{s}, r\right) .
\end{aligned}
$$


In the threshold limit, $\tilde{D}_{a b}$ can be written in terms of the higher-order function $\omega_{a b}\left(n, \alpha_{s}\right)$ for the invariant-mass distribution discussed above. The resummation may then be performed as before. Details will be presented elsewhere. In Fig. 1(right), we show the $K$-factor for the Drell-Yan rapidity distribution in the J-PARC experiment, at NLO and for the resummed case. The $K$-factors increase toward larger $\eta$, since one approaches the threshold regime more closely there.

\section{Summary}

We have discussed higher-order pQCD effects in the mass and rapidity distributions for the Drell-Yan process at the proposed GSI and J-PARC experiments. The corrections are very large, but seem under control when the soft-gluon resummation is implemented. We hope that our studies, along with the complementary study for transverse-momentum distributions ${ }^{15}$, will be of use in comparisons to future data from the GSI and J-PARC.

\section{Acknowledgments}

We are grateful to H. Shimizu and G. Sterman for collaboration on some of the work described here. H.Y.'s work is supported in part by a Research Fellowship of the Japan Society for the Promotion of Science. W.V. is supported by DOE Contract No. DE-AC02-98CH10886.

\section{References}

1. V. Barone et al. [PAX Collaboration], arXiv:hep-ex/0505054; M. Maggiora et al. [ASSIA Collaboration], arXiv:hep-ex/0504011.

2. D. Dutta et al., Letter of Intent for "Physics of High-Mass Dimuon Production at the 50-GeV Proton Synchrotron"; S. Sawada, these proceedings.

3. H. Shimizu, G. Sterman, W. Vogelsang and H. Yokoya, Phys. Rev. D 71, 114007 (2005); Nucl. Phys. Proc. Suppl. 157, 197 (2006).

4. G. Sterman, Nucl. Phys. B 281, 310 (1987); S. Catani and L. Trentadue, Nucl. Phys. B 327, 323 (1989); ibid. 353, 183 (1991).

5. R. Hamberg et al., Nucl. Phys. B 359, 343 (1991); ibid. B 644, 403 (2002).

6. W. Vogelsang, Phys. Rev. D 57, 1886 (1998).

7. A. Vogt, Phys. Lett. B 497, 228 (2001); S. Catani et al., JHEP 0307, 028 (2003); S. Moch and A. Vogt, Phys. Lett. B 631, 48 (2005).

8. T. O. Eynck, E. Laenen and L. Magnea, JHEP 0306, 057 (2003).

9. S. Catani et al., Nucl. Phys. B 478, 273 (1996).

10. M. Kramer, E. Laenen and M. Spira, Nucl. Phys. B 511, 523 (1998); S. Catani, D. de Florian and M. Grazzini, JHEP 0201, 015 (2002). 
11. A. Kulesza, G. Sterman and W. Vogelsang, Phys. Rev. D 66, 014011 (2002).

12. C. Anastasiou et al., Phys. Rev. D 69, 094008 (2004).

13. O. Martin et al., Phys. Rev. D 60, 117502 (1999).

14. G. Sterman and W. Vogelsang, JHEP 0102, 016 (2001);

A. Mukherjee and W. Vogelsang, Phys. Rev. D 73, 074005 (2006).

15. H. Kawamura et al., Prog. Theor. Phys. 115, 667 (2006); these proceedings. 